\title{
Influence of ecological filters on phytoplankton communities in semi-arid solar saltern environments
}

\author{
Influência dos filtros ecológicos nas comunidades fitoplanctônicas em ambientes de \\ salinas solares no semiárido
}

Raiane Santos Costa ${ }^{1}$, Joseline Molozzi ${ }^{1}$, Luiz Ubiratan Hepp ${ }^{2}$, Diógenes Félix da Silva Costa ${ }^{3}$,

Paulo Roberto Nunes da Silva ${ }^{1}$, Renato de Medeiros Rocha ${ }^{3}$ and José Etham de Lucena Barbosa ${ }^{1}$

${ }^{1}$ Laboratório de Ecologia Aquática, Laboratório de Ecologia de Bentos, Programa de Pós-graduação em Ecologia e Conservaçấo, Departamento de Biologia, Universidade Estadual da Paraíba - UEPB, Av. das Bananeiras, 351, CEP 58109-753, Campina Grande, PB, Brazil

e-mail: raianegeo@hotmail.com; jmolozzi@gmail.com; paulobiologo13@gmail.com; ethambarbosa@ hotmail.com

${ }^{2}$ Departamento de Ciências Biológicas, Universidade Regional Integrada do Alto Uruguai e das Missóes URI, Campus de Erechim, Av. Sete de Setembro, 1621, CEP 99700-000, Erechim, RS, Brazil e-mail: luizuhepp@gmail.com

${ }^{3}$ Departamento de Geografia, Universidade Federal do Rio Grande do Norte - UFRN, Rua José Evaristo, CEP 59300-000, Caicó, RN, Brazil e-mail: diogenesgeo@yahoo.com.br; renatocaico@yahoo.com.br

\begin{abstract}
Objective: This study analyzed the influence of ecological filters (abiotic variables) on the phytoplankton community in hypersaline ecosystems. Methods: The abiotic variables measured herein were: $\mathrm{pH}$, water temperature, salinity, ammonium ion, total nitrogen, nitrite, nitrate, total phosphorus, and soluble reactive phosphorus. The phytoplankton community was studied for density, richness and Shannon-Wiener diversity index. Data were analyzed using analysis of variance, linear regression and Canonical correspondence analysis (CCA). Results: In total, 110 taxa were identified in 3 solar salterns, distributed into 9 taxonomic classes, with the predominance of Cyanobacteria (41.8\%) and Bacillariophyceae (22.7\%). The species Pseudanabaena galeata was the only species sampled in all saline environments. Salinity significantly influenced the richness in the three salterns, Augusto Severo $\left(\mathrm{F}_{(1,22)}=23.2, \mathrm{p}<0.001\right)$, Francisco Menescal $\left(\mathrm{F}_{(1,22)}=50.02, \mathrm{p}<0.001\right)$ and Santa Clara $\left(\mathrm{F}_{(1,22)}=66.33, \mathrm{p}<0.001\right)$. The first two CCA axes explained $41.6 \%$ of total data variability, with a negative relationship with soluble reactive phosphorus for axis 2 . Conclusion: The study showed the influence of the dynamics of salterns ecosystems on the phytoplankton community structure. This is associated with filters developed by the environment, where the increasing salinity, temperature and precipitation of salts influence the composition of these organisms in the ecosystem.
\end{abstract}

Keywords: environments hypersaline; salinity; algae; abiotic variables; richness.

Resumo: Objetivo: Este trabalho visou analisar a influência dos filtros ecológicos (variáveis abióticas) na comunidade fitoplanctônica nos ecossistemas hipersalinos. Métodos: As variáveis abióticas mensuradas no estudo foram: $\mathrm{pH}$, temperatura da água, salinidade, íons amônio, nitrogênio total, nitrito, nitrato, fósforo total, fósforo reativo solúvel. A comunidade fitoplanctônica foi estudada em nível de riqueza, densidade, Diversidade de Shannon-Wiener. Os dados foram tratados através de análise de variância, regressão linear e uma análise de correspondência canônica (ACC). Resultados: No total foram identificados 110 taxa nas 3 salinas solares, distribuídos em 9 classes taxonômicas, havendo o predomínio das classes Cyanobacteria (41,8\%), Bacillariophyceae $(22,7 \%)$. A espécie Pseudanabaena galeata foi à única espécie amostrada em todos os ambientes salinos. A salinidade influenciou significativamente na riqueza nas três Salinas, Augusto Severo $\left(\mathrm{F}_{(1,22)}=23,2 ; \mathrm{p}<0,001\right)$, Francisco Menescal $\left(\mathrm{F}_{(1,22)}=50,02 ; \mathrm{p}<0,001\right)$ e Santa Clara $\left(\mathrm{F}_{(1,22)}=66,33 ; \mathrm{p}<0,001\right)$. Na análise de correspondência canônica (ACC) os dois primeiros eixos explicaram $41,6 \%$ da variabilidade total dos dados, tendo apenas relação negativa com o fósforo reativo solúvel para o eixo 2. Conclusáo: $\mathrm{O}$ estudo evidenciou a 
influência da dinâmica do ecossistema salino na estrutura da comunidade fitoplanctônica. Esse fato está associado aos filtros desenvolvidos pelo ambiente, onde o aumento crescente da salinidade, da temperatura, da precipitação dos sais, acaba influenciando na composição desses organismos no ecossistema.

Palavras-chave: ambientes hipersalinos; salinidade; algas; variáveis abióticas; riqueza.

\section{Introduction}

Wetlands are among the most important ecosystems in the world (Cardoso et al., 2012). These systems are considered unique owing to their hydrology and role as ecotone between terrestrial and aquatic ecosystems (Mitsch \& Gosselink, 2007). Scientifically, however, little is known about these systems, mainly concerning hypersaline wetlands, which need to be studied at the level of the dynamics of ecological and economic processes and hydrogeochemical characteristics (De Medeiros Rocha et al., 2012; Costa et al., 2014).

Solar salterns are artificial ecosystems located in tropical and subtropical regions of the world, consisting of a series of interconnected evaporators (evaporators and crystallizers), in which sea or estuarine water is abstracted and transferred from one pond to another by gravity or electrical pumping (Davis, 2000; Abid et al., 2008). These environments have cultural, scientific, historical and commercial values for the local society and worldwide societies (De Medeiros Rocha et al., 2012). Sola salterns are considered wetlands due to the ecological services provided, such as artisanal fisheries, refuge zones for migratory birds, great biological diversity and the presence of endemic species of hypersaline environments (PedrósAlió et al., 2000; Costa et al., 2014).

These hypersaline environments show different compositions of the planktonic flora because of geographic, climatic, physical, chemical and biological factors (Williams, 1998), which work as ecological filters by selecting species that cannot develop in the environment (Weiher \& Keddy, 1995; Poff, 1997). Species selection occurs when biotic and abiotic factors limit the development of species (Poff, 1997). Biological filters are related to ecological interactions (competition and predation) that ultimately influence the dispersion in the local diversity. Moreover, abiotic filters are associated with species constraints, which can only develop or adapt under certain environmental conditions (Myers \& Harms, 2009).

Forbes (1887) was one of the first authors to address this relationship between biological characteristics and environmental conditions. Since then, many other authors have advanced the research on the influence of ecological filters on the development of species in ecosystems (Statzner et al., 2001; Heino et al., 2003; Bonada et al., 2005; Myers \& Harms, 2009; Roque et al., 2010). Thus, environmental factors began to consider filters which select species that fail to develop in the conditions of existing habitats (Tonn, 1990, Heino et al., 2003, Townsend et al., 2003).

In the biological system of salterns, planktonic organisms influence the production of sea salt and the maintenance of the ecological status of the brine (Davis, 1978, 2009; Oren et al., 2009). The communities present in the environment are adapted to different salinity ranges, and algae play a different role in each sector (Oren et al., 2009), such as the control of mucilage, nutrient cycling, and balance of trophic levels, as well as other functions that are of great importance in maintaining the balance of the ecosystem along the salterns circuit (Davis, 2000).

The microorganisms that make up this environment are interconnected through the food chain, with phytoplankton at the bottom of this web (Reynolds, 2006). The instability of the hypersaline system, relative to the salinity gradient and changing environmental conditions throughout the system, determine phytoplankton composition, with patterns of species richness, diversity and density directly related to the dynamics of salterns (Pedrós-Alió et al., 2000). According to Thiéry \& Puente (2002), in their study in a saline water body in France, environmental factors are considered inhibitors of the growth of some species, especially salinity and temperature, both of which are abiotic factors that are considered filter inhibitors of species from one sector to the other.

In this context, this study aimed to analyze the influence of ecological filters (abiotic variables) on the phytoplankton community in hypersaline ecosystems. We hypothesized that environmental variables (salinity and temperature) are predictors in the selection and inhibition of species that do not tolerate high salt concentrations, but there are 
halotolerant or halophilic species that can thrive in the extreme environment of salterns.

\section{Materials and Methods}

\subsection{Study area}

The study was conducted in three salterns of the Brazilian semiarid region (Figure 1), located on the margins of the Apodi-Mossoró River estuary, northern coast of Rio Grande do Norte State, Brazil. Which is characterized by a longitudinal gradient of salinity from the mouth and along the estuary, with a progressive increase in salinity; this averages $30 \pm 50$ g. $\mathrm{L}^{-1}$ in the upper section of the estuary, and this dynamics of salinity characterizes it as a negative or hypersaline estuary (Medeiros et al., 2010).

The first saltern, Augusto Severo (145.08 ha), is located at the mouth of the estuary of the ApodiMossoró River (0457'54”S and 3708'29”W), in the municipality of Areia Branca/RN, and pumps water directly from the sea $\left(30 \pm 35\right.$ g. $\left.\mathrm{L}^{-1}\right)$. The second saltern, Francisco Menescal (4.000 ha), is located approximately $10 \mathrm{~km}$ from the mouth of the estuary, in the municipality of Mossoró/RN ( $5^{\circ} 3^{\prime} 53^{\prime \prime} \mathrm{S}$ and $\left.37^{\circ} 11^{\prime} 29^{\prime \prime} \mathrm{W}\right)$, abstracting water directly from the estuary $\left(40 \pm 50 \mathrm{~g} . \mathrm{L}^{-1}\right)$. The last saltern, the Santa Clara (1.045 ha), is located $30 \mathrm{~km}$ from the mouth on the margin of the estuary, in the municipality of Mossoró/RN (5\%6'18"S and $\left.37^{\circ} 15^{\prime} 10^{\prime \prime} \mathrm{W}\right)$, also pumping directly from the estuary $\left(60 \pm 70\right.$ g. $\left.\mathrm{L}^{-1}\right)$.

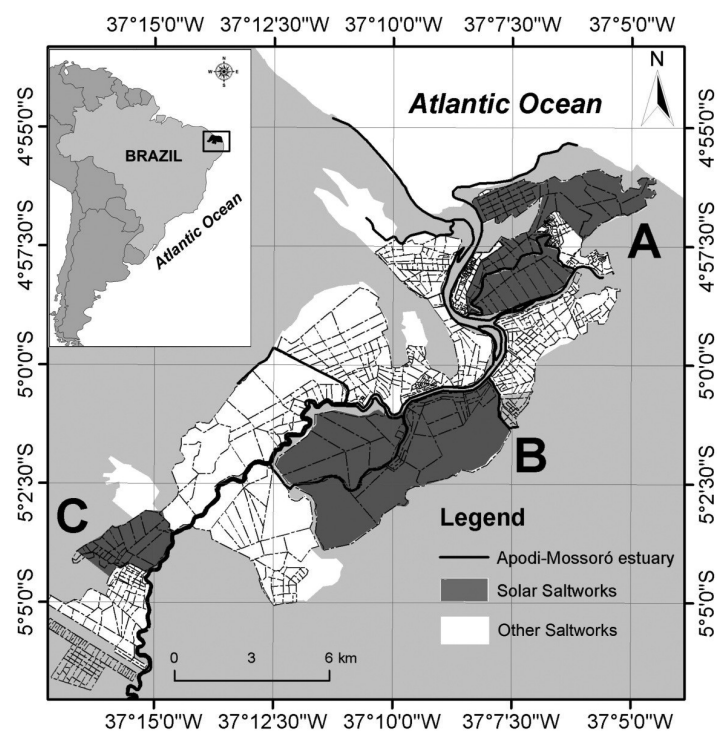

Figure 1. Location of the hypersaline wetlands (Salterns Augusto Severo (A), Menescal Francisco (B) and Santa Clara (C)) on the Apodi-Mossoró River, Rio Grande do Norte State.

\subsection{Sampling}

Sampling was performed in January 2013 in three salterns (Figure 2). Due to the salinity, the saline gradient was divided into 4 sectors (Initial Sector (35-90 g. $\left.\mathrm{L}^{-1}\right)$, Intermediate Sectors I and II, (100-190 g. $\left.\mathrm{L}^{-1}\right)$ Final Sector (200-250 g. $\left.\mathrm{L}^{-1}\right)$ and Crystallizers (250-300 g. $\left.\mathrm{L}^{-1}\right)$ ), which consisted of a series of interconnected evaporators that are, in turn, the compartments that form the sectors (Davis, 2000).

Samplings of the phytoplankton community and limnological variables were carried out in the Initial, Intermediate I and II sectors for each salt. In situ, 2 evaporators were chosen from each sector (the first and the last with different salinity) for sampling the plankton community. Thus, 6 evaporators at each saltern were sampled, from which 4 samples were taken, averaging 24 samples per saltern, and totaling 72 samples in the 3 studied salterns.

\subsection{Environmental variables}

Water temperature was measured in situ with a thermometer (TermistoTenmars TM 744R RS-232), and salinity with a portable digital refractometer (Fischer). The $\mathrm{pH}$ was measured by a Hanna digital pH meter (HI9224). Analyses of ammonium ions, total nitrogen, nitrite, nitrate, total phosphorus and soluble reactive phosphorus were performed according to APHA et al. (1998).

\subsection{Biological data}

Phytoplankton samples were taken at the subsurface with a plankton net $(20 \mu \mathrm{m})$ by filtering 200 liters for each sample, which were placed in polyethylene vials $(50 \mathrm{~mL})$ and preserved in $4 \%$ formaldehyde solution for qualitative analysis. The quantitative analysis of phytoplankton was obtained by counting $2 \mathrm{~mL}$ of the sample in a SedgewickRafter chamber (Chellappa et al., 2008, 2009). The result was multiplied by the volume of the analyzed sample $(50 \mathrm{~mL})$ then divided by the volume filtered $\left(200,000 \mathrm{~mL}^{-1}\right)$, thereby obtaining the density (ind. $\mathrm{mL}^{-1}$ ) of individuals. According to the method of

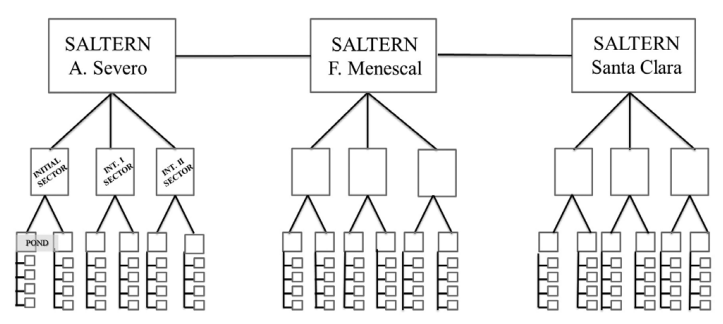

Figure 2. Sampling design of the studied salterns (Augusto Severo, Francisco Mescal and Santa Clara). 
Chellappa et al. (2008, 2009), the count of the individuals was used, because it uses the qualitative sample to determine phytoplankton density.

Identification of organisms was performed using a Zeiss binocular microscope, with up to 1,000 times magnification. Taxa were identified with the aid of taxonomic keys, whenever possible to specific and infra-specific levels. For species identification, we used specific literature: Cupp (1943); Uherkovich (1966); Komárek \& Anagnostidis (1988); Anagnostidis \& Komárek (1988); Hegewald \& Silva (1988); Tenenbaum et al. (2004); Metzeltin et al. (2005); and Tucci et al. (2012).

\subsection{Data analysis}

The frequency of occurrence of phytoplankton was determined based on the data of species richness. Organisms were classified into: rare species $(\leq 20 \%)$, infrequent species $(>20 \% \leq 50 \%)$, frequent species $(>50 \% \leq 80 \%)$ and very frequent species (> 80\%), following the method proposed by Mateucci \& Colma (1982). Species diversity was calculated based on the Shannon-Wiener Diversity index (Shannon, 1948).

Along the saltern system, the existence of ecological filters was predicted, promoted by the salt gradient and environmental conditions. For the analyses to show the influence of the filters, we considered sampling in each saltern compartment, diversity among sectors (Initial Sector, Intermediate I and II Sectors) and the geographical location of the salterns on the hypersaline estuary.

In order to check for differences between species richness and limnological variables, a oneway ANOVA was run for each variable (Gotelli \& Ellison, 2011). A simple linear regression was employed to determine the influence of the salinity gradient in each studied salterns on the species richness. Canonical Correspondence Analysis (CCA) was applied to detect relationships between density of phytoplankton species and environmental variables analyzed in the three hypersaline zones. All of these analyses were run using the vegan package (Oksanen et al., 2012) in R software (R Core Team, 2012).

\section{Results}

\subsection{Biotic characterization}

In total, 110 taxa were identified (Table 1), distributed into 9 taxonomic classes: Cyanobacteria $(41.8 \%)$, Bacillariophyceae $(22.7 \%)$, Coscinodiscophyceae (16.4\%), Chlorophyceae (6.3\%), Trebouxiophyceae (3.6\%), Fragilariophyceae (3.6\%), Euglenophyceae (3.6\%), Dinophyceae

Table 1. Phytoplankton taxa and frequency of occurrence of species in Augusto Severo, Francisco Menescal and Santa Clara salterns. $\mathrm{I}=$ initial sector, $\mathrm{II}=$ intermediate sector, III $=$ intermediate sector. FO = Frequency of occurrence, $\mathrm{R}=$ rare species $(\leq 20 \%), \mathrm{PF}=$ infrequent species $(>20 \% \leq 50 \%)$.

\begin{tabular}{|c|c|c|c|c|c|c|c|c|c|c|}
\hline \multirow[t]{2}{*}{ Species } & \multicolumn{3}{|c|}{$\begin{array}{l}\text { Augusto } \\
\text { Severo }\end{array}$} & \multicolumn{3}{|c|}{$\begin{array}{l}\text { Francisco } \\
\text { Menescal }\end{array}$} & \multicolumn{3}{|c|}{ Santa Clara } & \multirow[t]{2}{*}{ FO } \\
\hline & $\mathbf{I}$ & II & III & $\mathbf{I}$ & II & III & $\mathbf{I}$ & II & III & \\
\hline \multicolumn{11}{|l|}{ Bacillariophyceae } \\
\hline Actinoptychus Ehrenberg, 1843 & $x$ & $\mathrm{x}$ & - & - & - & - & - & - & - & $\mathrm{R}$ \\
\hline Amphiprora alata (Ehrenberg) Kützing, 1844 & - & - & - & $x$ & - & - & - & - & - & $\mathrm{R}$ \\
\hline Amphora Ehrenberg ex Kützing, 1844 & $x$ & - & - & $x$ & - & - & $x$ & - & - & $\mathrm{R}$ \\
\hline Amphora veneta Kützing, 1844 & $x$ & - & - & $x$ & - & - & - & - & - & $\mathrm{R}$ \\
\hline Caloneis sp. Cleve, 1894 & $\mathrm{x}$ & - & - & - & - & - & - & - & - & $\mathrm{R}$ \\
\hline Cerataulina pelagica (Cleve) Hendey, 1937 & $\mathrm{x}$ & - & - & - & - & - & $\mathrm{x}$ & - & - & $\mathrm{R}$ \\
\hline Coscinodiscus curvatulus Ehrenberg, 1839 & $x$ & $x$ & - & - & - & $x$ & - & $x$ & - & PF \\
\hline Coscinodiscus Ehrenberg, 1839 & - & $x$ & - & - & $x$ & - & $x$ & $x$ & - & PF \\
\hline Coscinodiscus excentricus Ehrenberg, 1840 & - & - & - & $x$ & - & - & - & - & - & $\mathrm{R}$ \\
\hline Craticula sp. Grunow, 1868 & $x$ & $x$ & $x$ & - & $x$ & $x$ & - & $x$ & $x$ & PF \\
\hline Cyclotella meneghiniana Kützing, 1844 & - & $\mathrm{x}$ & - & - & - & - & - & - & - & $\mathrm{R}$ \\
\hline $\begin{array}{l}\text { Dactyliosolen fragilissimus (Bergon) Hasle in Hasle \& Syvertsen, } \\
1996\end{array}$ & - & - & - & - & - & - & - & - & $\mathrm{x}$ & $\mathrm{R}$ \\
\hline Fragilaria sp. Müller, 1819 & $\mathrm{x}$ & - & - & $\mathrm{x}$ & - & - & - & - & - & $\mathrm{R}$ \\
\hline Fragilaria bidens Heiberg, 1863 & $\mathrm{x}$ & - & - & - & - & - & - & - & - & $\mathrm{R}$ \\
\hline Fragilaria capucina Desmazières, 1830 & $\mathrm{x}$ & - & - & - & - & - & $\mathrm{x}$ & - & - & $\mathrm{R}$ \\
\hline Fragilaria intermedia (Grunow) Grunow in van Heurck, 1881 & - & - & - & - & $x$ & - & - & - & - & $\mathrm{R}$ \\
\hline Fragilaria intermedia (Grunow) Grunow in van Heurck, 1881 & - & - & - & - & $\mathrm{x}$ & - & - & - & - & $\mathrm{R}$ \\
\hline Geissleria Lange-Bertalot \& Metzeltin, 1996 & - & $\mathrm{x}$ & - & - & $\mathrm{x}$ & $x$ & $\mathrm{x}$ & - & - & PF \\
\hline Gyrosigma Hassall, 1845 & - & - & - & - & - & - & - & $x$ & - & $\mathrm{R}$ \\
\hline
\end{tabular}


Table 1. Continued...

\begin{tabular}{|c|c|c|c|c|c|c|c|c|c|c|}
\hline \multirow[t]{2}{*}{ Species } & \multicolumn{3}{|c|}{$\begin{array}{l}\text { Augusto } \\
\text { Severo }\end{array}$} & \multicolumn{3}{|c|}{$\begin{array}{l}\text { Francisco } \\
\text { Menescal }\end{array}$} & \multicolumn{3}{|c|}{ Santa Clara } & \multirow[t]{2}{*}{ FO } \\
\hline & I & II & III & $\mathbf{I}$ & II & III & $\mathbf{I}$ & II & III & \\
\hline Gyrosigma obscurum (W.Smith) J.W.Griffith \& Henfrey, 1856 & $\mathrm{x}$ & - & - & - & - & - & - & $\mathrm{x}$ & - & $\mathrm{R}$ \\
\hline Mastogloia sp. Thwaites ex W.Smith, 1856 & - & - & - & - & $\mathrm{x}$ & - & - & - & - & $\mathrm{R}$ \\
\hline Melosira sp. C. Agardh, 1824 & $\mathrm{x}$ & - & - & - & $\mathrm{x}$ & - & $\mathrm{x}$ & - & - & $\mathrm{R}$ \\
\hline Navicula sp. Bory de Saint-Vincent, 1822 & $\mathrm{x}$ & - & - & - & - & - & - & - & - & $\mathrm{R}$ \\
\hline Nitzschia closterium (Ehrenberg) W. Smith, 1853 & - & - & - & - & - & - & $\mathrm{x}$ & - & - & $\mathrm{R}$ \\
\hline Nitzschia dissipata (Kützing) Grunow, 1862 & $\mathrm{x}$ & - & - & - & - & - & $\mathrm{x}$ & - & - & $\mathrm{R}$ \\
\hline Nitzschia Hassall, 1845 & - & - & - & $\mathrm{x}$ & - & - & $\mathrm{x}$ & - & - & $\mathrm{R}$ \\
\hline Nitzschia longissima (Brébisson) Ralfs in Pritchard, 1861 & $\mathrm{x}$ & - & - & - & - & - & $\mathrm{x}$ & - & - & $\mathrm{R}$ \\
\hline Nitzschia palea (Kützing) W.Smith, 1856 & $\mathrm{x}$ & - & - & $\mathrm{x}$ & - & - & - & - & - & $\mathrm{R}$ \\
\hline $\begin{array}{l}\text { Nitzschia paradoxa (J.F.Gmelin) Grunow in Cleve \& Grunow, } \\
1880\end{array}$ & - & - & - & - & - & - & $x$ & - & - & $\mathrm{R}$ \\
\hline Nitzschia sigma (Kützing) W.Smith, 1853 & - & $\mathrm{x}$ & - & - & - & - & - & - & - & $\mathrm{R}$ \\
\hline Paralia sulcata (Ehrenberg) Cleve, 1873 & - & $\mathrm{x}$ & $\mathrm{x}$ & - & - & - & - & - & - & $\mathrm{R}$ \\
\hline Pinnularia sp. Ehrenberg, 1843 & - & $\mathrm{x}$ & - & - & - & - & - & - & - & $\mathrm{R}$ \\
\hline Pleurosigma sp. W.Smith, 1852 & $\mathrm{x}$ & - & - & $x$ & - & - & $\mathrm{x}$ & - & - & $\mathrm{R}$ \\
\hline Rhizosolenia sp. Brightwell, 1858 & - & - & $\mathrm{x}$ & - & - & - & - & - & - & $\mathrm{R}$ \\
\hline Surirella fastuosa Ehrenberg, 1859 & $x$ & - & - & - & - & - & - & - & - & $\mathrm{R}$ \\
\hline Surirella ovata Kützing, 1844 & $x$ & $x$ & - & $x$ & $x$ & - & - & - & - & PF \\
\hline Surirella splendida (Ehrenberg) Kützing, 1844 & $\mathrm{x}$ & - & - & - & $\mathrm{x}$ & $\mathrm{x}$ & $\mathrm{x}$ & $\mathrm{x}$ & - & PF \\
\hline Surirella sp. Turpin, 1828 & $\mathrm{x}$ & - & - & $\mathrm{x}$ & $\mathrm{x}$ & - & - & - & - & $\mathrm{R}$ \\
\hline Thalassiosira aestivalis Gran in Gran \& Angst, 1931 & - & - & - & $x$ & - & - & - & - & - & $\mathrm{R}$ \\
\hline Thalassiosira Cleve, 1873 & - & - & - & - & - & - & - & - & $x$ & $\mathrm{R}$ \\
\hline Thalassiosira decipiens (Grunow) E.G.Jørgensen, 1905 & - & - & - & - & $x$ & - & - & - & - & $\mathrm{R}$ \\
\hline \multicolumn{11}{|l|}{ Coscinodiscophyceae } \\
\hline $\begin{array}{l}\text { Biddulphia mobiliensis (J.W.Bailey) } \\
\text { Grunow, } 1882\end{array}$ & $\mathrm{x}$ & - & - & - & - & - & - & - & - & $\mathrm{R}$ \\
\hline Guinardia delicatula (Cleve) Hasle in Hasle \& Syvertsen, 1997 & - & - & - & $x$ & - & - & - & - & - & $\mathrm{R}$ \\
\hline Guinardia flaccida (Castracane) H.Peragallo, 1892 & $x$ & - & $x$ & - & - & - & $x$ & - & - & $\mathrm{R}$ \\
\hline Guinardia sp. H. Peragallo, 1892 & - & - & - & $x$ & - & - & - & - & - & $\mathrm{R}$ \\
\hline Hemidiscus cuneiformis Wallich, 1860 & $x$ & - & - & - & - & - & - & - & $x$ & $\mathrm{R}$ \\
\hline Leptocylindrus danicus Cleve, 1889 & $x$ & - & - & - & - & - & - & - & - & $\mathrm{R}$ \\
\hline \multicolumn{11}{|l|}{ Cyanophyceae } \\
\hline $\begin{array}{l}\text { Aphanizomenon A.Morren ex Bornet \& } \\
\text { Flahault, } 1888\end{array}$ & $x$ & - & - & - & - & - & - & - & $x$ & $\mathrm{R}$ \\
\hline Aphanocapsa annulata G.B.McGregor, 2004 & $x$ & - & - & - & $x$ & - & - & - & - & $\mathrm{R}$ \\
\hline Aphanocapsa elachista West \& G.S.West, 1894 & $x$ & - & - & - & - & - & - & - & - & $\mathrm{R}$ \\
\hline Aphanothece conglomerata Rich, 1932 & - & - & - & - & - & - & $x$ & $\mathrm{x}$ & $x$ & $\mathrm{R}$ \\
\hline Aphanothece pallida (Kützing) Rabenhorst, 1863 & $x$ & - & - & - & - & - & - & $x$ & $x$ & $\mathrm{R}$ \\
\hline Arthrospira platensis (Nordstedt) Gomont, 1892 & - & - & - & - & $x$ & $x$ & - & - & - & $\mathrm{R}$ \\
\hline Borzia sp. Cohn ex Gomont, 1892 & $x$ & - & - & $x$ & - & - & $x$ & - & - & $\mathrm{R}$ \\
\hline Borzia susedana Ercegovic, 1925 & - & - & - & $x$ & - & - & $x$ & - & - & $\mathrm{R}$ \\
\hline Chroococcus giganteus West, 1892 & $x$ & - & - & - & $x$ & - & - & - & - & $\mathrm{R}$ \\
\hline Chroococcus sp. Nägeli, 1849 & - & - & - & - & - & - & $x$ & - & - & $\mathrm{R}$ \\
\hline Chroococcus turgidus (Kützing) Nägeli, 1849 & $x$ & $x$ & - & $x$ & $x$ & - & - & - & - & PF \\
\hline $\begin{array}{l}\text { Coelomoron tropicale P. A. C. Senna, A. C. Peres \& Komárek, } \\
1998\end{array}$ & $x$ & - & - & $x$ & - & - & - & - & - & $\mathrm{R}$ \\
\hline $\begin{array}{l}\text { Coleofasciculus chthonoplastes (Gomont) } \\
\text { M.Siegesmund, J.R.Johansen \& T.Friedl in Siegesmund et al., } \\
2008\end{array}$ & $x$ & - & - & $\mathrm{x}$ & $x$ & - & - & - & - & $\mathrm{R}$ \\
\hline Gloeothece sp. Nägeli, 1849 & - & - & - & - & $\mathrm{x}$ & - & - & - & - & $\mathrm{R}$ \\
\hline Gomphosphaeria aponina Kützing, 1836 & - & - & - & $x$ & - & - & - & - & - & $\mathrm{R}$ \\
\hline Lyngbya aestuarii Liebman ex Gomont, 1892 & $x$ & - & - & - & - & - & - & - & - & $\mathrm{R}$ \\
\hline Lyngbya majuscula (Dillwyn) Harvey, 1833 & $x$ & - & - & - & - & - & - & - & - & $\mathrm{R}$ \\
\hline Merismopedia Meyen, 1839 & - & - & - & $\mathrm{x}$ & - & - & - & - & - & $\mathrm{R}$ \\
\hline $\begin{array}{l}\text { Microcystis panniformis J.Komárek, J.Komárková-Legnerová, } \\
\text { C.L.Sant'Anna, M.T.P.Azevedo, \& P.A.C.Senna, } 2002\end{array}$ & $x$ & - & - & - & $x$ & - & - & - & - & $\mathrm{R}$ \\
\hline
\end{tabular}


Table 1. Continued...

\begin{tabular}{|c|c|c|c|c|c|c|c|c|c|c|}
\hline \multirow[t]{2}{*}{ Species } & \multicolumn{3}{|c|}{$\begin{array}{l}\text { Augusto } \\
\text { Severo }\end{array}$} & \multicolumn{3}{|c|}{$\begin{array}{l}\text { Francisco } \\
\text { Menescal }\end{array}$} & \multicolumn{3}{|c|}{ Santa Clara } & \multirow[t]{2}{*}{ FO } \\
\hline & I & II & III & $\mathbf{I}$ & II & III & $\mathbf{I}$ & II & III & \\
\hline $\begin{array}{l}\text { Microcystis wesenbergii (Komárek) Komárek in Kondrat'eva, } \\
1968\end{array}$ & - & - & $\mathrm{x}$ & - & - & - & - & $\mathrm{x}$ & - & $\mathrm{R}$ \\
\hline Odontella mobiliensis (J.W.Bailey) Grunow, 1884 & $\mathrm{x}$ & $\mathrm{x}$ & - & - & $\mathrm{x}$ & $\mathrm{x}$ & - & $\mathrm{x}$ & $\mathrm{x}$ & PF \\
\hline Oscillatoria curviceps C. Agardh, 1824 & - & - & - & $x$ & $\mathrm{x}$ & - & $\mathrm{x}$ & - & - & $\mathrm{R}$ \\
\hline Oscillatoria formosa Bory de Saint-Vincent ex Gomont, 1892 & $\mathrm{x}$ & - & - & - & - & $\mathrm{x}$ & $\mathrm{x}$ & $\mathrm{x}$ & $\mathrm{x}$ & PF \\
\hline Oscillatoria limosa C.Agardh, 1812 & - & - & - & $x$ & $\mathrm{x}$ & - & - & - & - & $\mathrm{R}$ \\
\hline Oscillatoria sancta Kützing ex Gomont, 1892 & $\mathrm{x}$ & - & - & $x$ & - & - & - & - & - & $\mathrm{R}$ \\
\hline Oscillatoria tenuis C.Agardh, 1813 & - & $x$ & - & $x$ & $x$ & - & - & - & - & $\mathrm{R}$ \\
\hline Oscillatoria princeps Vaucher ex Gomont, 1803 & - & $\mathrm{x}$ & - & $\mathrm{x}$ & $\mathrm{x}$ & - & $\mathrm{x}$ & $\mathrm{x}$ & - & PF \\
\hline Oscillatoria sp. Vaucher ex Gomont, 1892 & $\mathrm{x}$ & - & - & - & - & - & - & - & - & PF \\
\hline Phormidium sp. Kützing ex Gomont, 1892 & $\mathrm{x}$ & $\mathrm{x}$ & - & - & $x$ & - & $x$ & $\mathrm{x}$ & $\mathrm{x}$ & PF \\
\hline Phormidium tenue (Meneghini) Gomont, 1892 & - & - & $x$ & - & - & - & - & - & - & $\mathrm{R}$ \\
\hline $\begin{array}{l}\text { Phormidium tergestinum (Rabenhorst ex Gomont) Anagnostidis } \\
\text { \& Komárek, } 1988\end{array}$ & - & - & - & - & - & - & $\mathrm{x}$ & - & - & $\mathrm{R}$ \\
\hline Planktothrix agardhii (Gomont) Anagnostidis \& Komárek, 1988 & $\mathrm{x}$ & - & - & $\mathrm{x}$ & $\mathrm{x}$ & $\mathrm{x}$ & $\mathrm{x}$ & $\mathrm{x}$ & $\mathrm{x}$ & PF \\
\hline Planktothrix isothrix (Skuja) Komárek \& Komárková, 2004 & - & - & - & $x$ & - & - & $\mathrm{x}$ & - & - & $\mathrm{R}$ \\
\hline Pseudanabaena catenata Lauterborn, 1915 & $\mathrm{x}$ & $\mathrm{x}$ & $\mathrm{x}$ & $\mathrm{x}$ & $\mathrm{x}$ & $\mathrm{x}$ & $\mathrm{x}$ & $\mathrm{x}$ & $\mathrm{x}$ & PF \\
\hline Pseudanabaena galeata Böcher, 1949 & $\mathrm{x}$ & - & - & $\mathrm{x}$ & $\mathrm{x}$ & - & - & - & - & $\mathrm{R}$ \\
\hline Pseudanabaena Lauterborn, 1915 & $\mathrm{x}$ & - & - & $\mathrm{x}$ & - & - & - & - & - & $\mathrm{R}$ \\
\hline Pseudanabaena limnetica (Lemmermann) Komárek, 1974 & - & - & $x$ & - & - & - & $\mathrm{x}$ & - & - & $\mathrm{R}$ \\
\hline Sphaerocavum sp. M.T. de P.Azevedo \& C.L.Sant'Anna, 2003 & $\mathrm{x}$ & - & - & $\mathrm{x}$ & - & - & - & - & - & $\mathrm{R}$ \\
\hline Spirulina labyrinthiformis Gomont, 1892 & - & - & - & - & - & - & $\mathrm{x}$ & - & - & $\mathrm{R}$ \\
\hline Spirulina laxissima G.S.West, 1907 & - & - & - & - & $x$ & - & - & - & - & $\mathrm{R}$ \\
\hline Spirulina major Kützing, 1843 & - & - & - & $x$ & - & - & - & - & - & $\mathrm{R}$ \\
\hline Spirulina subsalsa Oersted, 1842 & $x$ & - & - & - & - & - & - & - & - & $\mathrm{R}$ \\
\hline Peridinium furca Ehrenberg, 1834 & - & - & - & - & - & - & $x$ & - & - & $\mathrm{R}$ \\
\hline \multicolumn{11}{|l|}{ Euglenophyceae } \\
\hline Euglena Ehrenberg, 1830 & - & - & - & $x$ & - & - & $x$ & - & - & $\mathrm{R}$ \\
\hline Euglena spirogyra Ehrenberg, 1832 & - & - & - & $x$ & - & - & - & - & - & $\mathrm{R}$ \\
\hline Trachelomonas armata var. armata (Ehrenberg) Stei, 1835 & $\mathrm{x}$ & - & - & - & - & - & $\mathrm{x}$ & - & - & $\mathrm{R}$ \\
\hline Trachelomonas Ehrenberg, 1835 & - & - & - & - & - & - & $\mathrm{x}$ & - & - & $\mathrm{R}$ \\
\hline \multicolumn{11}{|l|}{ Zygnemophyceae } \\
\hline Cosmarium Corda ex Ralfs, 1848 & - & - & - & $x$ & - & - & $x$ & - & - & $\mathrm{R}$ \\
\hline
\end{tabular}

(1\%), Conjugatophyceae (1\%). The predominance of Cyanobacteria and Bacillariophyceae indicate their adaptation to the saline environment. Seventytwo taxa were found at the Augusto Severo Saltern, with the predominance of Cyanobacteria and Bacillariophyta (36 taxa and 22 taxa, respectively), 64 taxa at the Francisco Menescal Saltern, with predominance of Cyanobacteria and Bacillariophyta (41 taxa and 27 taxa, respectively) and 45 taxa at the Santa Clara Saltern, with a greater abundance of Cyanobacteria and Bacillariophyta (33 taxa and 15 taxa, respectively).

Pseudanabaena galeata (Böcher) was the only species sampled in all of the saline environments. Around 96 taxa were rare species distributed in the three salterns (Table 1). The phytoplanktonic density was 0.203 ind. $\mathrm{mL}^{-1}, 0.481$ ind. $\mathrm{mL}^{-1}$ and 0.333 ind. $\mathrm{mL}^{-1}$, respectively, for the salterns
Augusto Severo, Francisco Menescal and Santa Clara (Table 2). The values of Shannon-Wiener diversity index were similar between Francisco Augusto and Severo Menescal salterns (0.098 and 0.097 , respectively) and about 2.5 times lower than the Santa Clara salterns.

The species richness decreased significantly with increasing salt concentration $\left(\mathrm{F}_{(1,70)}=63.52\right.$; $\mathrm{p}<0.001$ ) (Figure 3). On average, a loss of species occurred with an increase of about $17 \mathrm{mg} . \mathrm{L}^{-1}$ salt. Salinity significantly influenced the richness in the three salterns (Figure 3), Augusto Severo $\left(\mathrm{F}_{(1,22)}=23.2 ; \mathrm{p}<0.001\right)$, Francisco Menescal $\left(\mathrm{F}_{(1,22)}=50.02 ; \mathrm{p}<0.001\right)$ and Santa Clara $\left(\mathrm{F}_{(1,22)}\right.$ $=66.33 ; \mathrm{p}<0.001)$.

In the CCA, the first two axes explained $41.6 \%$ of total data variability. Axis 1 explained $21 \%$ and axis 2 explained $20.6 \%$ (Figure $4 \mathrm{a}$ and $\mathrm{b}$ ); by Monte 
Table 2. Density (\%) of the most representative species of the hypersaline wetlands. I = initial sector, II = intermediate sector, III = intermediate sector. $(-)=$ absence of species.

\begin{tabular}{|c|c|c|c|c|c|c|c|c|c|}
\hline \multirow[t]{2}{*}{ Species } & \multicolumn{3}{|c|}{ Augusto Severo } & \multicolumn{3}{|c|}{$\begin{array}{l}\text { Francisco } \\
\text { Menescal }\end{array}$} & \multicolumn{3}{|c|}{ Santa Clara } \\
\hline & I & II & III & $\mathrm{I}$ & II & III & I & II & III \\
\hline \multicolumn{10}{|l|}{ Bacillariophyta } \\
\hline Amphiprora Ehrenberg, 1843 & - & - & - & - & - & - & 23.48 & - & - \\
\hline Amphora veneta Kützing 1844 & 1.6 & - & - & - & - & - & - & - & - \\
\hline $\begin{array}{l}\text { Aphanizomenon A. Morren ex Bornet \& Flahault, } \\
1888\end{array}$ & 5.8 & - & - & - & - & - & 31.55 & - & - \\
\hline Cerataulina pelagica (Cleve) Hendey 1937 & - & - & - & 0.65 & 0.09 & - & - & - & - \\
\hline Fragilaria capucina Desmazières 1830 & 0.9 & - & - & - & - & - & - & - & - \\
\hline Fragilaria Lyngbye, 1819 & 0.12 & - & - & - & - & - & - & - & - \\
\hline Gyrosigma Hassall, 1845 & 12.51 & - & - & 14.45 & - & - & - & - & - \\
\hline $\begin{array}{l}\text { Gyrosigma obscurum (W.Smith) J.W.Griffith \& } \\
\text { Henfrey } 1856\end{array}$ & 2.19 & - & - & - & - & - & - & - & - \\
\hline Mastogloia Thwaites ex W.Smith, 1856 & - & - & - & 0.15 & 20.45 & - & - & - & - \\
\hline Navicula Bory de Saint-Vincent, 1822 & 38.06 & 40.42 & - & 7.7 & - & - & - & - & - \\
\hline Nitzschia closterium (Ehrenberg) W. Smith 1853 & 7.09 & - & - & 76.01 & - & - & - & - & - \\
\hline Nitzschia dissipata (Kützing) Grunow 1862 & 0.25 & - & - & - & - & - & - & - & - \\
\hline Nitzschia Hassall, 1845 & 10.45 & - & - & 0.32 & - & - & - & - & - \\
\hline Nitzschia sigma (Kützing) W. Smith 1853 & 5.67 & - & - & - & - & - & - & - & - \\
\hline Pleurosigma W. Smith, 1852 & 0.51 & - & - & - & - & - & - & - & - \\
\hline Surirella tuberosa Otto Müller 2006 & 0.51 & - & - & 0.07 & - & - & - & - & - \\
\hline \multicolumn{10}{|l|}{ Cyanophyta } \\
\hline Aphanocapsa annulata G.B. McGregor 2004 & 5.29 & - & - & - & - & - & - & - & - \\
\hline Aphanothece conglomerata Rich 1932 & 5.16 & - & - & - & - & - & - & 98.8 & 99.05 \\
\hline Aphanothece pallida (Kützing) Rabenhorst 1863 & 1.8 & 54.69 & 90.9 & - & 79.08 & 100 & - & - & - \\
\hline \multicolumn{10}{|l|}{ Coscinodiscophyta } \\
\hline Biddulphia mobiliensis (J.W. Bailey) Grunow 1882 & 0.12 & - & - & - & - & - & - & - & - \\
\hline Hemidiscus cuneiformis Wallich 1860 & 0.38 & - & - & - & - & - & - & - & - \\
\hline \multicolumn{10}{|l|}{ Chlorophyta } \\
\hline Protoperidinium simulum (Paulsen) Balech 1974 & - & - & - & - & - & - & 3.3 & - & - \\
\hline $\begin{array}{l}\text { Scrippsiella trochoidea (Stein) Balech ex Loeblich III } \\
1965\end{array}$ & - & - & 9.09 & - & - & - & 14.68 & - & - \\
\hline \multicolumn{10}{|l|}{ Euglenophyta } \\
\hline Trachelomonas Ehrenberg, 1835 & - & - & - & 0.15 & - & - & - & - & - \\
\hline
\end{tabular}

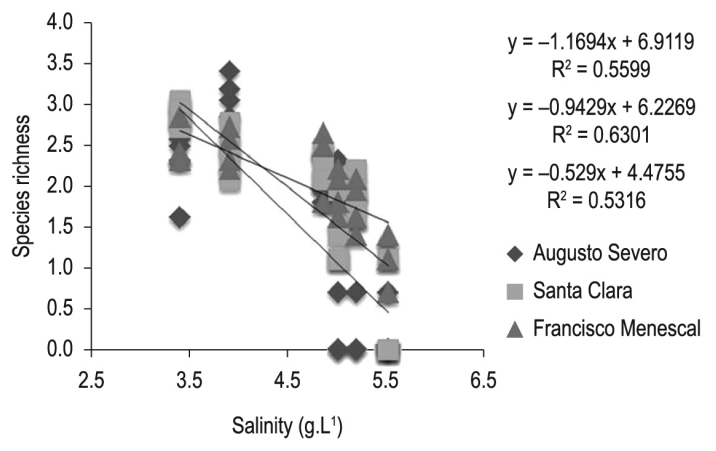

Figure 3. Effects of salinity on algal richness in the salterns (Augusto Severo, Francisco Menescal and Santa Clara).

Carlo analysis of the CCA test, this is significant $(\mathrm{p}=0.03)$. In axis 1 , abiotic variables (salinity, temperature, nitrogen and nitrite) showed positive correlations with the sampling sites located in the two last sectors (Sectors Intermediate I and II) of the three salterns. In axis 2, nitrite, ammonium, total phosphorus and soluble reactive phosphorus presented positive correlations with the sampling sites located on the Initial Sector, except for the second pond of Francisco Menescal and Augusto Severo salterns, which were negatively correlated with soluble reactive phosphorus in the same axis. The species Aphanothese pallida (Apa) and Mastogoia sp. (Mas) showed a positive correlation with axis 2 , and negative correlation to axis 1 ; since the species Gyrosigma sp. (Gyr), Nitzschia sigma (Nsi), Nitzschia sp. (Nit), and Nitzschia closterium $(\mathrm{Ncl})$ have a positive correlation with axis 1 , there was a negative correlation with axis 2. Species Amphiprora sp. (Amp), Aphanizomenon sp (Aph), Chroococus turgidus (Ctu), Lyngbya majuscul (Lma), Oscillatória sancta (Osa), Phormidium sp. (Pho), Protoperidiniu 
(a)

(b)
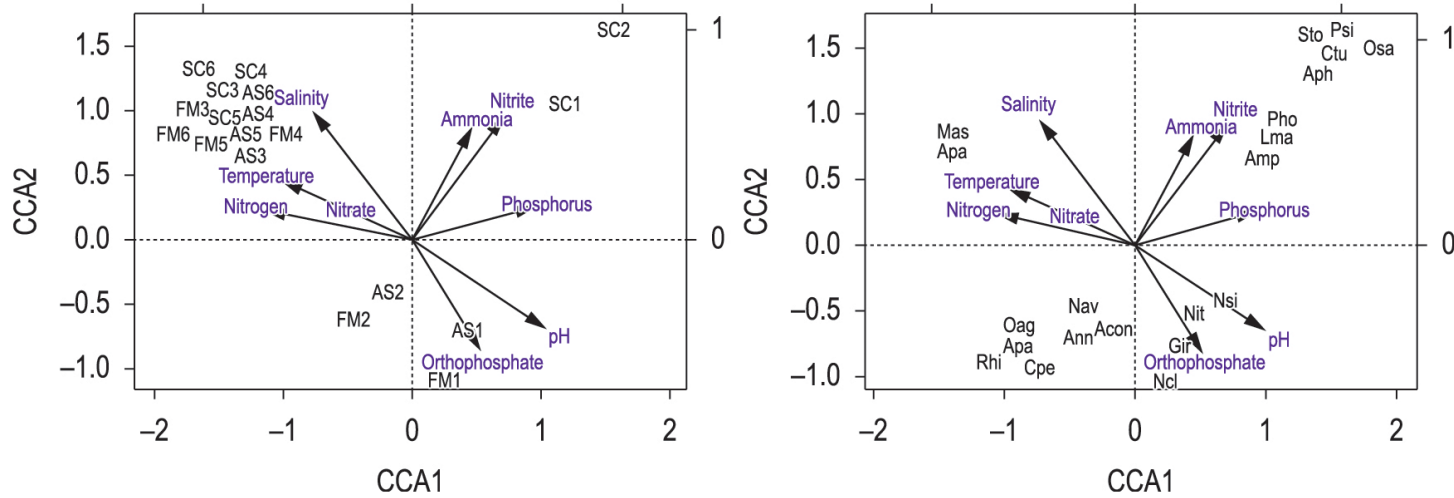

Figure 4. Canonical Correspondence Analysis (CCA) between the main phytoplankton species and environmental variables in Augusto Severo (AS), Francisco Menescal (FM) and Santa Clara (SC) salterns in the semi-arid region; evaporators (1 to 6); Sectors: Initial Sector (evaporator 1 and 2); Intermediate Section I (3:04 evaporator) and Sector Intermediate (5:06 evaporator). (a): Corresevaporators to abiotic variables in relation to sampled sites. (b): Corresevaporators to abiotic variables in relation to phytoplankton species. Legend: Amp: Amphiprora sp.; Aph: Aphanizomenon sp.; Ann: Aphanocapsa annulata; Acon: Aphanothece conglomerata; Apa: Aphanothece pallida; Ctu: Chroococus turgidus; Gyr: Gyrosigma sp.; Lma: Lyngbya majuscula; Mas: Mastogoia sp.; Nav: Navicula sp.; Ncl: Nitzschia closterium; Nsi: Nitzschia sigma; Nit: Nitzschia sp.; Ncl: Nitzschia closterium; Osa: Oscillatoria sancta; Pho: Phormidium sp.; Psi: Protoperidinium simulum; Sto: Scrippsiella tochoidea; Oag: Oscillatoria agardhii; Cpe: Cerataulina pelagica; Tra: Trachelomonas sp. and Rhi: Rhizosolenia sp.

sumulum (Psi) and Scripsiella tochoidea (Sto) showed a positive correlation with the 2 axes of the CCA.

\section{Discussion}

In the present study, the phytoplankton communities were influenced by ecological filters, which were characterized as being abiotic conditions existing in the environment. The gradient of salinity and temperature in particular are the environmental variables that most influence the richness and abundance of species, as evidenced in the study by Thiéry \& Puente (2002). However, along the hypersaline ecosystem, abiotic variables and the interaction between species become ecological filters, inhibiting the development of species.

The abiotic conditions of each sector selected organisms that do not have biological characteristics that allow them to survive, develop and reproduce in increasingly restrictive environments (Townsend \& Hildrew, 1994; Statzner et al., 2001). This effect was evidenced by Abid et al. (2008), who showed that physical and chemical parameters (salinity, $\mathrm{pH}$, temperature, total phosphorus, soluble reactive phosphorus, total nitrogen, nitrate, nitrite, and total dissolved salts), as well as the geographical location of the saltern, influence the decrease in species richness.

The passage of the biological community through the sectors will result in the selection of species that are better adapted to local environmental conditions, as observed in this study by the prevalence of two groups of classes, Cyanobacteria and Bacillariophyceae, and the development of a single species (Pseudanabaena galeata) along the salt gradient; i.e. they had managed to overcome the ecological filters by adapting to environmental conditions. To overcome a filter, a species requires physiological characteristics (Poff, 1997) such as osmotic balance, nitrification, sulfate reduction with the formation of methane acetate, and processes of respiration, photosynthesis and fermentation (Oren, 2002).

Several authors (e.g. Pedrós-Alió et al., 2000; Oren, 2002; Ayadi et al., 2004), in their studies on phytoplankton communities and the microbial web in solar saltworks developed in Israel, Tunisia and Spain, respectively, have evidenced the influence of hypersaline ecosystem dynamics, such as abiotic factors, especially salinity and temperature, as a selective filter of species richness and density, influencing the structure of the biological community; these groups observed species that are able to develop along the salt gradient. Nevertheless, there is a loss of the pool of local species in each sector of the saltern, which was also observed in the present study, where the Initial Sector had greater richness and density of species, with a decrease in species richness and density along 
with the movement of brine to the other sectors (Sectors Intermediate I and II). This was observed in the regression analysis, and in the scatter plot for the three salterns.

The ecological filters developed in this environment led to the selection of many organisms and the emergence of new species adapted to such conditions (Oren, 2001). Thus, biological diversity will eventually reduce depending on the filters developed by the environment, such as high salinity, physical and chemical conditions, and resource availability, thus affecting the diversity and structure of the phytoplankton community (Ayadi et al., 2004; Winder \& Sommer, 2012). As observed in the canonical correspondence analysis, the environmental variables ( $\mathrm{pH}$, total phosphorus, nitrogen, salinity and temperature) have a positive influence.

There was a clear positive influence $(\mathrm{pH}$, ammonium, total nitrogen, nitrite, nitrate and total phosphorus) on the density and spatial variation of the plankton community along the hypersaline ecosystem, except for soluble reactive phosphate, which has a negative influence for some phytoplankton communities. Similarly, in hypersaline and marine environments, some authors observed that the phytoplankton community was influenced by environmental variables (ammonia, nitrite, nitrate, phosphate, temperature, oxygen) and salinity gradient (Giordano et al., 1994; Pedrós-Alió et al., 2000; Thiéry \& Puente, 2002; Telesh et al., 2013). These abiotic factors inhibit the development of the pool of local species (Chalmandrier et al., 2013); thus, the species cannot develop to other sectors.

In the Shannon-Wiener index, the Santa Clara Saltern showed the highest value. Already in relation to richness, the Augusto Severo Saltern had the highest number of species identified, which is associated with its location in the estuary, and abstraction of water directly from the sea; hence, it has lower salinity levels in relation to the other salterns studied, thus contributing to the fact that it does not report great species loss. Regarding the phytoplankton density, the Francisco Menescal Saltern presented higher density, despite higher levels of salinity compared with the Augusto Severo Saltern. However, as evidenced by Pedrós-Alió et al. (2000) in two Spanish salterns, the increased salinity resulted in reduced abundance and a number of different groups of eukaryotic microorganisms, but an increase in biomass of prokaryotes. The significant reduction in species richness in the system was also verified by Thiéry \& Puente (2002) in the Camargue Saltern, France, which showed the influence of temperature and salt concentrations on the reduction of plankton abundance (Williams, 1998). Chalmandrier et al. (2013) observed the effects of biotic interactions (predation, competition, resources) on the community structure, but these biotic interactions are difficult to investigate due to the selective effect of abiotic variables. The decrease in species richness and diversity in the salterns is related to ecological filters imposed along the saline environment. Thus, this allows the appearance of species adapted to this environment, in this case, halotolerant and halophilic species (Williams, 1998; Oren, 2001).

The decrease in richness and diversity of species in salt, too, is due to the inverse dynamics of the estuary, i.e. increased salinity of the mouth towards the mainland, which provides a rich biological diversity directly related to the estuary dynamics (Telesh et al., 2013).

Thus, the importance of studies that address the effect of ecological filters on biological communities is noticed. This is in order to have a better understanding of ecosystem dynamics, making it possible to recommend measures for their conservation and management by taking into account the study of biological communities.

The present study evidenced the dynamics of the saline environment and its influence on the structure of phytoplankton communities. This fact is associated with filters developed by the hypersaline environment, where increasing salinity, temperature and precipitation of salts influence the reduction in the richness and density of these organisms in the ecosystem. Therefore, the hypothesis was confirmed, since the dynamics of the hypersaline ecosystem (abiotic factors) becomes a selective filter of species due to the unsuitable abiotic conditions for the development of species other than those which are halotolerant or halophilic.

\section{Acknowledgements}

The authors would like to thank salt companies: Comércio e Indústria Salineira (CIASAL), Salinas do Nordeste S/A (SALINOR) and Andrea Jales Rosado, which allowed the study, as well as all the support in the field. We also thank the financial support from the "Conselho Nacional de Desenvolvimento Científico e Tecnológico (CNPq) and the Coordenação de Aperfeiçoamento de Pessoal de Nível Superior (CAPES), Semi-Arid Ecology Laboratory (LABESA), which provided 
logistic support for field samplings. To the Limnology Laboratory and to Benthos Ecology Laboratory of the UEPB for help in the analysis and identification. LUH received financial support from FAPERGS (Process \#12/1354-0) and CNPq (Process \#471572/2012-8).

\section{References}

ABID, O., SELLAMI-KAMMOUN, A., AYADI, H., DRIRA, Z., BOUAIN, A. and ALEYA, L. Biochemical adaptation of phytoplankton to salinity and nutrient gradients in a coastal solar saltern, Tunisia. Estuarine, Coastal and Shelf Science, 2008, 80(3), 391-400. http://dx.doi.org/10.1016/j. ecss.2008.09.007.

AMERICAN PUBLIC HEALTH ASSOCIATION - APHA, AMERICAN WATER WORKS ASSOCIATION - AWWA and WATER ENVIRONMENT FEDERATION - WEF. Standard methods for the examination of water and wastewater. 20th ed. New York: APHA/AWWA/ WEF, 1998, $1220 \mathrm{p}$.

ANAGNOSTIDIS, K. and KOMÁREK, J. Modern approach to the classification system of cyanophytes. 3 - Oscillatoriales. Algological Studies/Archiv für Hydrobiologie, 1988, 50-53, 327-472.

AYADI, H., ABID, O., ELLOUMI, J., BOUAÏN, A. and SIME-NGANDO, T. Structure of the phytoplankton communities in two lagoons of different salinity in the Sfax saltern (Tunisia). Journal of Plankton Research, 2004, 26(6), 669-679. http:// dx.doi.org/10.1093/plankt/fbh047.

BONADA, N., ZAMORA-MUNOZZ, C., RIERADEVALL, M. and PRAT, N. Ecological and historical filters constraining spatial caddis fly distribution in Mediterranean rivers. Freshwater Biology, 2005, 50(5), 781-797. http://dx.doi. org/10.1111/j.1365-2427.2005.01357.x.

CARDOSO, S.J., ROLAND, F., LOVERDEOLIVEIRA, S.M. and HUSZAR, V.L.D.M. Phytoplankton abundance, biomass and diversity within and between Pantanal wetland habitats. Limnologica - Ecology and Management of Inland Waters, 2012, 42(3), 235-241. http://dx.doi. org/10.1016/j.limno.2012.01.002.

CHALMANDRIER, L., MÜNKEMÜLLER, T., GALLIEN, L., DE BELLO, F., MAZEL, F., LAVERGNE, S. and THUILLER, W. A family of null models to distinguish between environmental filtering and biotic interactions in functional diversity patterns. Journal of Vegetation Science, 2013, 24(5), 853-864. http://dx.doi.org/10.1111/jvs.12031. PMid:24791143

CHELLAPPA, N.T., BORBA, J.M. and ROCHA, O. Phytoplankton community and physical-chemical characteristics of water in the public reservoir of
Cruzeta, RN, Brazil. Brazilian Journal of Biology, 2008, 68(3), 477-494. http://dx.doi.org/10.1590/ S1519-69842008000300004. PMid:18833468

CHELLAPPA, N.T., CÂMARA, F.R.A. and ROCHA, O. Phytoplankton community: indicator of water quality in the Armando Ribeiro Gonçalves reservoir and Pataxó channel, Rio Grande do Norte, Brazil. Brazilian Journal of Biology, 2009, 69(2), 241-251. http://dx.doi.org/10.1590/S151969842009000200003. PMid:19675924

COSTA, D.F.S., DE MEDEIROS ROCHA, R., BARBOSA, J.E.L., SOARES, A.M.V.M. and LILLEBO, A.I. Análise dos serviços ambientais prestados pelas salinas solares. Board Game Geek, 2014, 41(1), 195-209.

CUPP, E.E. Marine plankton diatom of the West coast of North America. Bulletin of the Scripps Institution of Oceanography, 1943, 6(1), 1-238.

DAVIS, J.S. Biological communities of a nutrient enriched salina. Aquatic Botany, 1978, 4, 23-42. http://dx.doi.org/10.1016/0304-3770(78)90004-9.

DAVIS, J.S. Management of biological systems for continuously-operated solar saltworks. Global NEST Journal, 2009, 11(1), 73-78.

DAVIS, J.S. Structure, function, and management of the biological system for seasonal solar salt works. Global NEST Journal, 2000, 2(3), 217-226.

DE MEDEIROS ROCHA, R., COSTA, D.F.S., LUCENA-FILHO, M.A., BEZERRA, R.M., MEDEIROS, D.H.M., AZEVEDO-SILVA, A.M., ARAUJO, C.N. and XAVIER-FILHO, L. Brazilian solar saltworks-ancient uses and future possibilities. Aquatic Biosystems, 2012, 8(8), 2-6.

FORBES, S.A. The lake as a microcosm. Early Classics in Biogeography, Distribution and Diversity, 1887, 1-9.

GIORDANO, M., DAVIS, J.S. and BOWES, G. Organic carbon release by Dunaliella salina (Chlorophyta) under different growth conditions of CO2, nitrogen, and salinity. Journal of Phycology, 1994, 30(2), 249-257. http://dx.doi.org/10.1111/ j.0022-3646.1994.00249.x.

GOTElli, N.J. and ELlisON, A.M. Principios de estatística em Ecologia. Porto Alegre: Artmed, 2011, $528 \mathrm{p}$.

HEGEWALD, E. and SILVA, C.P. Annotated catalogue of scenedesmus and nomenclatural related genre, including original descriptions and figures. Berlim-Stuttgart: J. Cramer, 1988, $587 \mathrm{p}$.

HEINO, J., MUOTKA, T., MYKRÄ, H., PAAVOLA, R., HÄMÄLÄINEN, H. and KOSKENNIEMI, E. Defining macroinvertebrate assemblage types of headwater streams: implications for bioassessment and conservation. Ecological Applications, 2003, 13(3), 842-852. http://dx.doi.org/10.1890/10510761(2003)013[0842:DMATOH]2.0.CO;2. 
KOMÁREK, J. and ANAGNOSTIDIS, K. Modern approach to the classification system of Cyanophytes 4-Nostocales. Algological Studies/Archiv für Hydrobiologie, 1988, 56, 247-345.

MATEUCCI, S.D. and COLMA, A. La metodologia para el estudio de la vegetación. Washington, DC: Secretaría General de la Organización de los Estados Americanos, Programa Regional de Desarrollo Científico y Tecnológico, 1982, 168 p. Colleción de Monografias Cientificas, Série Biologia, no. 22.

MEDEIROS, A.M.A., BARBOSA, J.E.L., MEDEIROS, P.R., ROCHA, R.M. and SILVA, L.F. Salinity and freshwater discharge determine rotifer distribution at the Mossoró River Estuary (Semiarid Region of Brazil). Brazilian Journal of Biology, 2010, 70(3), 551-557. PMid:20730341.

METZELTIN, D., LANGE-BERTALOT, H. and GARCÍA-RODRIGUEZ, F. Diatoms of Uruguay: compared with other taxa from South America and elsewhere. Montevideo: A. R. G. Ganther, 2005, $736 \mathrm{p}$.

MITSCH, W.J. and GOSSELINK, J.G. Wetlands. 4th ed. New York: John Wiley and Sons, 2007, 582 p.

MYERS, J.A. and HARMS, K.E. Seed arrival, ecological filters, and plant species richness: a meta-analysis. Ecology Letters, 2009, 12(11), 1250-1260. http:// dx.doi.org/10.1111/j.1461-0248.2009.01373.x. PMid:19723285

OKSANEN, J., BLANCHET, F.G., KINDT, R., LEGENDRE, P., MINCHIN, P.R., O'HARA, R.B., SIMPSON, G.L. SOLYMOS, P., STEVENS, M.H.H. and WAGNER, H. 2012. vegan: Community Ecology Package. R package version 2.0-4 [online]. 2005 [viewed 10 Aug. 2013]. Available from: http:// CRAN.R-project.org/package=vegan

OREN, A. Halophilic microorganisms and their environments. Netherlands: Springer Netherlands, 2002, pp. 56-63.

OREN, A. The bioenergetic basis for the decrease in metabolic diversity in increasing salt concentrations: implications for the functioning of salt lake ecosystems. Hydrobiology, 2001, 466(1/3), 61-72. http://dx.doi.org/10.1023/A:1014557116838.

OREN, A., SØRENSEN, K.B., CANFIELD, D.E., TESKE, A.P., IONESCU, D., LIPSKI, A. and ALTENDORF, K. Microbial communities and processes within a hypersaline gypsum crust in a saltern evaporation pond (Eilat, Israel). Hydrobiology, 2009, 626(1), 15-26. http://dx.doi.org/10.1007/ s10750-009-9734-8.

PEDRÓS-ALIÓ, C., CALDERÓN-PAZ, J.I., MACLEAN, M.H., MEDINA, G., MARRASÉ, C., GASOL, J.M. and GUIXA-BOIXEREU, N. The microbial food web along salinity gradients. FEMS Microbiology Ecology, 2000, 32(2), 143-155.
http://dx.doi.org/10.1016/S0168-6496(00)00025-8. PMid:10817867

POFF, N.L. Landscape filters and species traits: towards mechanistic understanding and prediction in stream ecology. Journal of the American Benthological Norte Society, 1997, 16(2), 391-409. http://dx.doi. org/10.2307/1468026.

R CORE TEAM. $R$ : a Language and Environment for Statistical Computing [online]. Vienna: R Foundation for Statistical Computing, 2012 [viewed 10 Aug. 2014]. Available from: http://www.R-project.org

REYNOLDS, C.S. Ecology of phytoplankton. Cambridge: Cambridge University Press, 2006, 551 p.. http:// dx.doi.org/10.1017/CBO9780511542145.

ROQUE, F.O., SIQUEIRA, T., BINI, L.M., RIBEIRO, M.C., TAMBOSI, L.R., CIOCHETI, G. and TRIVINHO-STRIXINO, S. Untangling associations between chironomid taxa in Neotropical streams using local and landscape filters. Freshwater Biology, 2010, 55(4), 847-865. http://dx.doi. org/10.1111/j.1365-2427.2009.02314.x.

SHANNON, C.E. A mathematical theory of communication. Bulletin of System Tecnology Journal, 1948, 27(3), 379-423. http://dx.doi. org/10.1002/j.1538-7305.1948.tb01338.x.

STATZNER, B., BIS, B., DOLÉDEC, S. and USSEGLIO-POLATERA, P. Perspectives for biomonitoring at large spatial scales: a unified measure for the functional composition of invertebrate communities in European running waters. Basic and Applied Ecology, 2001, 2(1), 73-85. http://dx.doi. org/10.1078/1439-1791-00039.

TELESH, I., SCHUBERT, H. and SKARLATO, S. Life in the salinity gradient: Discovering mechanisms behind a new biodiversity pattern. Estuarine, Coastal and ShelfScience, 2013, 135, 317-327. http://dx.doi. org/10.1016/j.ecss.2013.10.013.

TENENBAUM, D.R., VILLAC, M.C., VIANNA, S.C., MATOS, M., HATHERLY, M., LIMA, I.V. and MENEZES, M. Phytoplankon atlas of Sepetiba Bay, Rio de Janeiro, Brazil. London: IMO, 2004, 132 p. GloBallast Monograph Series, no. 16.

THIÉRY, A. and PUENTE, L. Crustacean assemblage and environmental characteristics of a man-made solar saltwork in southern France, with emphasis on anostracan (Branchiopod) population dynamics. Hydrobiology, 2002, 486(1), 191-200. http://dx.doi. org/10.1023/A:1021354903052.

TOWNSEND, C.R. and HILDREW, A.G. Species traits in relation to a habitat templet for river systems. Freshwater Biology, 1994, 31(3), 265-275. http:// dx.doi.org/10.1111/j.1365-2427.1994.tb01740.x.

TONN, W.M. Climate change and fish communites: a conceptual framework. Transactions of the American Fisheries Society, 1990, 119(2), 337-352. http://dx.doi. 
org/10.1577/1548-8659(1990)119<0337:CCAFCA $>2.3 . \mathrm{CO} ; 2$.

TOWNSEND, C.R., DOLÉDEC, S., NORRIS, R., PEACOCK, K. and ARBUCKLE, C. The influence of scale and geography on relationships between stream community composition and landscape variables: description and prediction. Freshwater Biology, 2003, 48(5), 768-785. http://dx.doi. org/10.1046/j.1365-2427.2003.01043.x.

TUCCI, A., SANT'ANA, C.L., AZEVEDO, M.T.P., MELCHER, S.S., WERNER, V.R., MALONE, C.F.S., ROSSINI, E.F., JACINAVICIUS, F.R., HENTSCHKE, G.S., OSTI, J.A.S., SANTOS, K.R.S., GAMA JUNIOR, W.A., ROSAL, C. and ADAME, G. Atlas de cianobactérias e microalgas de águas continentais brasileiras. São Paulo: Instituto de Botânica, 2012, 17 p.
UHERKOVICH, G. Die Scenedesmus-Arten Ungarns. Budapest: Akadémiai Kiadó: Verlag der Ungarisch. Akad. der Wissenschaften, 1966, 173 p.

WEIHER, E. and KEDDY, P.A. The assembly of experimental wetland plant communities. Oikos, 1995, 73, 323-335.

WILLIAMS, W.D. Salinity as a determinant of the structure of biological communities in salt lakes. Hydrobiology, 1998, 381(1/3), 191-201. http:// dx.doi.org/10.1023/A:1003287826503.

WINDER, M. and SOMMER, U. Phytoplankton response to a changing climate. Hydrobiology, 2012, 698(1), 5-16. http://dx.doi.org/10.1007/s10750012-1149-2.

Received: 21 February 2014 Accepted: 15 December 2014 